\title{
Synthesis and Characterization of Cobalt Doped Lysine Carbon Nanotubes
}

\author{
S. K. Shailesh, A. K. Roy, and B. Tiwari
}

\begin{abstract}
Carbon nanotubes are fascinating electronic material with unique physical properties and many important potential applications. Due to various applications of CNTs it is necessary to develop new methods for the synthesis of CNTS. In this chemical method, complexes of transition metal Co (II) with amino acid present in egg albumin have been synthesized. The complex so formed, is analyzed on the basis of spectroscopic method using, IR and NMR spectra confirms the lysine cobalt metal ion complex.

The lysine cobalt metal ion complex is decomposed at higher temperature using muffle furnace to get metal carbon nanotubes. These metal carbon nanotubes are characterized using scanning probe instruments like AFM and DLS and XRD.
\end{abstract}

Index Terms-Co (II), albumin, albumin-metal-complex, IR, NMR, AFM, DLS, X-RD.

\section{INTRODUCTION}

Carbon nanotubes (CNTS) are allotropes of Carbon with a nanostructure. The length to diameter ratio of CNT's is up to $28,000,000: 1$, which is significantly larger than any other material.

As a matter of fact CNT's are molecular scale tubes of graphitic carbon with outstanding properties. They are among the stiffest and strongest fibres known and have remarkable electronic properties with many other unique characteristic.

The carbon nanotube structure has already made it appearance because it represents an entirely new form of matter, Single-walled nanotubes can be either semiconductor or metallic. Nanotubes are also very stiff and very stable. They are built with their length exceeding thickness thousands of times.

Cluster of $\mathrm{C}$-atoms in cylindrical forms (carbon nanotubes) have novel properties that make them useful in many application in nanotechnology, electronics, optics, and other fields of materials science as well as potential uses in architectural fields.

Due to vast application of CNTs, it is required to develop new method and technique to prepare carbon nanotubes and characteristic them. It is also required to inculcate some desired properties in them, so that they can be used in various applications [1]-[3].

Manuscript received May 5, 2013; revised July 10, 2013

S. K. Shailesh is with the Dep. of Chemistry, Mewar University, Chittorgarh (Raj.), India (e-mail:skshailesh07@gmail.com).

A. K. Roy was with Indian school of mines, Dhanbad, India (e-mail: akr1906@gmail.com).

B. Tiwari is with the D.S. Institute of Technology and Management, Ghaziabad (UP), India (e-mail: tiwari.balendra@ rediffmail.com).

\section{SYNTHESIS}

\section{A. Chemical Method}

To prepare carbon nanotubes 0.5 normal $\mathrm{Co}^{2+}$ metal salt solutions was prepared in ethyl alcohol by AR grade chemicals. Then it is allowed to react with egg albumin, to form lysine-cobalt metal ion complex. The Cobalt-lysine complex so formed was decomposed at higher temperature as reported in the literature. It can be prepared in two steps:

\section{B. Preparation of Cobalt Lysine Complex}

As we know that proteins are macromolecules comprising of amino acid as monomer. Amino acids are compounds containing- $\mathrm{NH}_{2}$ and $\mathrm{COOH}$. With the help of these groups lysine form complexes with metals and different chains of amino acids combined together. These compounds on decomposition give carbon metal nanotubes.

When aqueous solution of cobalt salt is allowed to react with lysine present in egg albumin the lone pair present on nitrogen of $-\mathrm{NH}_{2}$ and oxygen of $\mathrm{COO}^{-}$of $\mathrm{COOH}$ group present in amino acid from complex with Co (II). In this was cobalt (II) forms cross links between two amino acid chains.

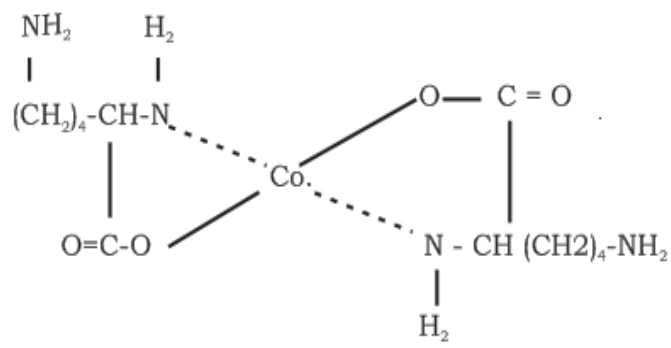

Fig. 1. Lysine-Cobalt (II) complex.

Egg albumin contains lysine $\left(\mathrm{NH}_{2} \quad\left(\mathrm{CH}_{2}\right)_{4} \quad \mathrm{CH}\right.$ $\left.\left(\mathrm{NH}_{2}\right) \cdot \mathrm{COOH}\right)$ amino acids. They react||with cobalt metal solution to give the following complexes (Fig. 1).

\section{Prepration of CNT}

In order to get CNT's the complex was decomposed in muffle furnace. The decomposition takes place in $800^{\circ} \mathrm{C}$, $900^{\circ} \mathrm{C}$ and $1000^{\circ} \mathrm{C}$ [4]-[6]. Multi-walled CNT's are prepared mostly at temperature as high as $800-900^{\circ} \mathrm{C}$. As at high temperature than this range the formation tends to single walled structure. The characterizations of multi-walled CNT's are done from AFM, DLS, X-RD, explained in the next section of this paper.

\section{CHARACTERIZATION}

\section{A. Spectral Studies}

Though it is very difficult to analyze the complexes of albumin and metal but certain important feature can be 
identified which give valuable information about the structure.

\section{1) IR spectra}

IR spectra of Cobalt lysine complex is shown in the Fig. 2. The information which we get by the IR Spectra is

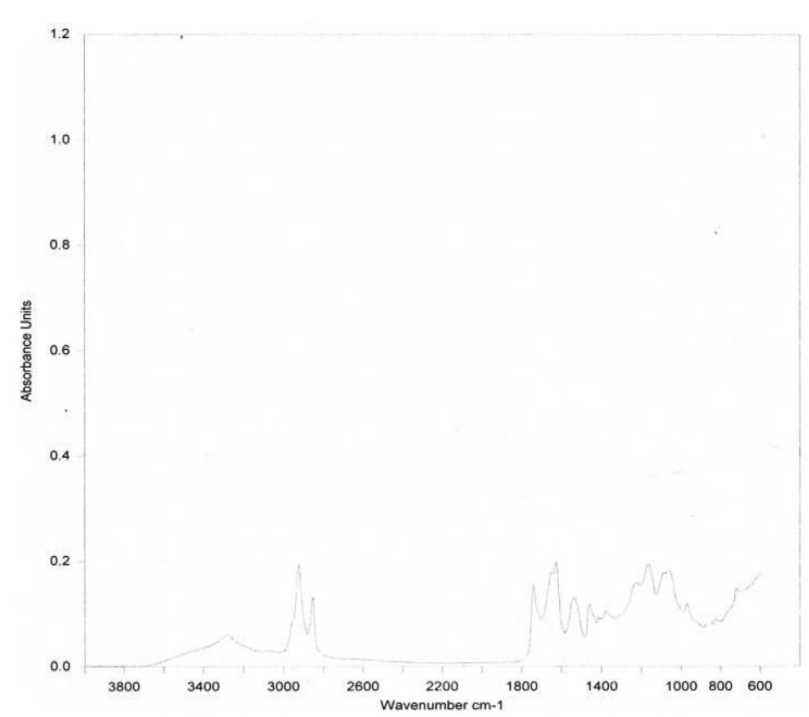

Fig. 2. IR Spectra of cobalt lysine complex.

In infrared spectroscopy, a weak band around the 3200 $\mathrm{cm}^{-1}$ assigned to the $\mathrm{N}-\mathrm{H}$ stretching vibration. $\mathrm{C}=\mathrm{O}$ stretching strong signal in amino acid occurs at the 1730$1755 \mathrm{~cm}^{-1}$. The strong intense bands at $1600 \mathrm{~cm}^{-1}$ of metal amino acid complex can be attributed to $\mathrm{NH}_{2}$ or $\mathrm{NH}_{3}{ }^{+}$ asymmetric bending and also a strong intense band at 1481$1550 \mathrm{~cm}^{-1}$ is assigned to the $\mathrm{NH}_{2}$ or $\mathrm{NH}_{3}^{+}$symmetric bending. The bands at $1400 \mathrm{~cm}^{-1}$ of metal amino acid complex indicates the presence of weak $\mathrm{C}=\mathrm{O}$ symmetric stretching in amino acid. The band at $1100 \mathrm{~cm}^{-1}$ can be assigned to the $\mathrm{C}-\mathrm{O}$ stretching in cobalt amino acid complex.

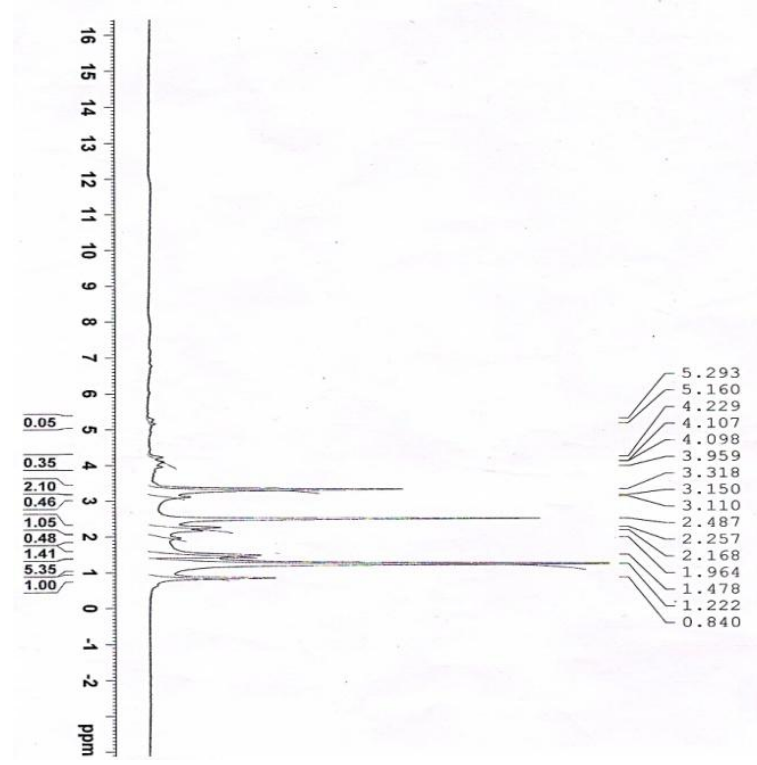

Fig. 3. 1H-NMR spectra of lysine cobalt metal ion complex.

\section{2) NMR spectra}

In the "H NMR spectra of the synthesized cobalt Amino acid Complex with chemical shifts within the range 3.318
PPM the $\mathrm{NH}_{2}$ Protons was observed (Fig. 3).

In the spectra, signal with chemical shift at 2.487 PPM C$\mathrm{H}$ Protons attached to the $\mathrm{COOH}$ in the amino acids was observed in the spectra signal with chemical shifts at 1.222 PPM is related to the $\mathrm{R}_{2} \mathrm{CH}_{2}$ (secondary type) Protons and the signal with chemical shift with the 0.840 PPM corresponds to the $\mathrm{R}^{\mathrm{C}} \mathrm{CH}_{3}$ (Primary type) protons also observed.

\section{B. Characterization by Scanning Probe Instrument \\ 1) Atomic Forces Microscopy (AFM)}

Surface imaging studies were performed using Atomic force microscopy to estimates surface morphology and particle size distribution. The samples were characterized at INSTITUTE OF INSTRUMENTATION CENTER, IIT ROORKEE. By this investigation we came to know that the linear dendritic shape of metal ions presents at the surface of Carbon nanotubes. The white spots in Fig. 4 show the presence of Co metal ions in rare linear dendritic form with mutli-walled structures.

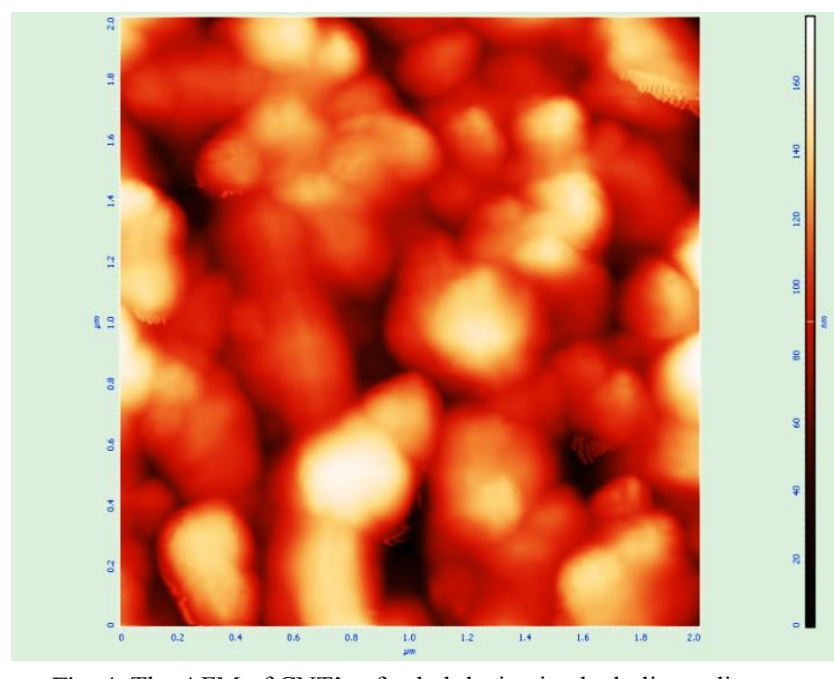

Fig. 4. The AFM of CNT's of cobalt lysine in alcoholic medium, decomposed at the $10000 \mathrm{C}$.

The amplitude or Height Parameters are the principal parameters in characterizing the surface topography. The average roughness $(R a)$ and the root mean square roughness $(R q)$ are the most used amplitude parameters. Particularly, the last one is used to study temporal changes in the creation of a new surface as well as spatial differences when studying the surface feature using different scales. The reason for this parameter is more sensitive to large deviations with respect to the mean line. To know if the $R q$ values are making sense, the height points have to be uniformly distributed according to a normal distribution curve [7]. If the height point distribution is not following a normal distribution, other statistical distribution could be attempted for determining the roughness if that is making any sense. The particle size predicted is $0-344.20 \mathrm{~nm}$ in diameter. The maximum peak to peak distance observed is $344.202 \mathrm{~nm}$ [8], [9]. Ten point heights is $179 \mathrm{~nm}$. Average sizes of peaks are $203.605 \mathrm{~nm}$ or Defined line distanced from the mean line (zi) with roughness approximately $37.0621 \mathrm{~nm}$.

\section{2) Dynamic Light Scattering (DLS)}

Dynamic light scattering techniques will give an intensity 
weighted distribution, where the contribution of each particle in the distribution relates to the intensity of light scattered by the particle.

It also measures the time dependent fluctuation in the scattering intensity to determine the translational diffusion coefficient $\left(\mathrm{D}_{\mathrm{T}}\right)$ and subsequently hydrodynamic radius $\left(\mathrm{R}_{\mathrm{H}}\right)$.

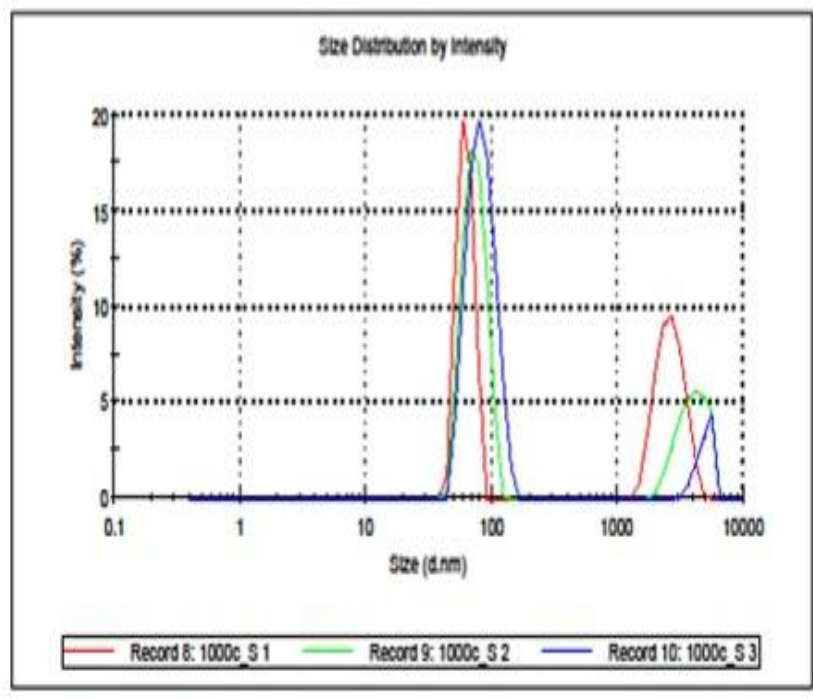

Fig. 5. DLS of the CNTs.

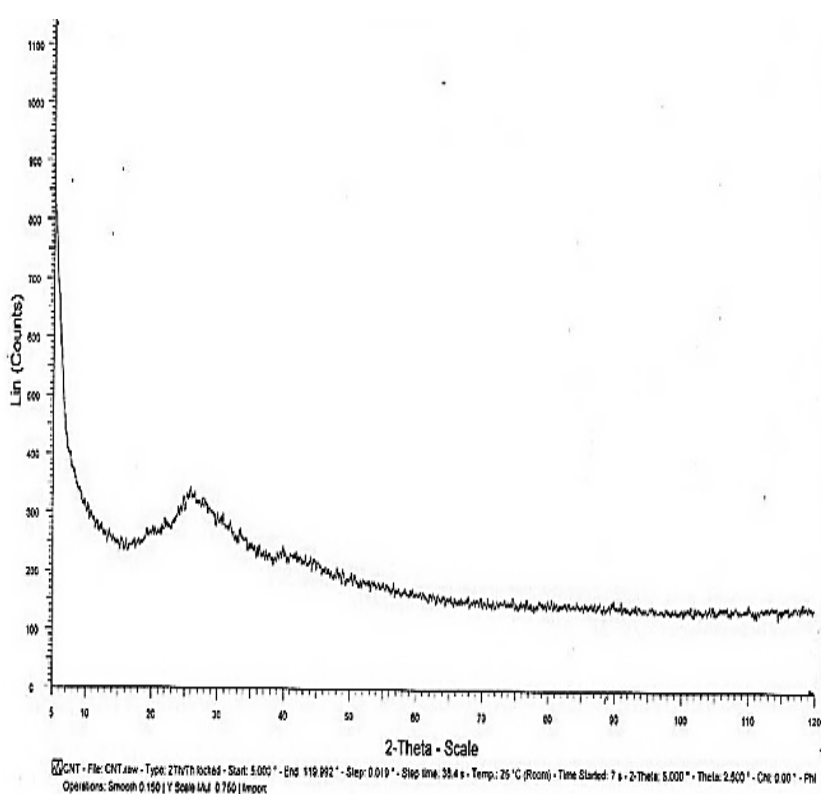

Fig. 6. X-RD of Cobalt doped Lysine carbon Nanotube.

Z-analysis or cumulate mean from the dynamic light scattering comes out to be $99.03 \mathrm{~nm}$. The Bimodal curves for each colored lines shows presence of impurities of $\mathrm{Co}$ ion complex and the polydespersity index value is 0.329 which shows broad size distribution of Co ion composites. The y-intercept can be used to evaluate the signal-to-noise ratio from a measured sample and thus is often used to judge data quality, the intercept value is 0.939 which shows a very good system as an ideal system gives value of 1.0. The three curves in the Fig. 5 shows number, volume and intensity distribution respectively.

\section{Characterization by $X-R D$}

The XRD peaks in the wide angle range of $2 \theta\left(20^{\circ}<2 \theta<\right.$ $90^{\circ}$ ) ascertained that the peaks in $24.662^{\circ}, 48.877^{\circ}, 50.208^{\circ}$, $69.804^{\circ}, 48.158^{\circ}$, can be attributed to the $3.606,1.8619$, 1.81560 , 1.34636, crystalline structures of lysine synthesized cobalt carbon nanotubes respectively.

The crystallite size can be determined by the Scherrer's formula is the wavelength of $\mathrm{X}$-ray radiation $(\mathrm{CuK} \alpha=$ $0.15406 \mathrm{~nm}$ ), is a constant taken as $0.89, \beta$ is the line width at half maximum height (FWHM) of the peak, and $\theta$ is the diffracting angle. The (101) plane was chosen to calculate the crystalline size (either plane can be used for this purpose). The average crystalline size for the CNTs synthesized and using lysine Solution confirms the particles size is approximately 11 to $18 \mathrm{~nm}$ respectively shows in the Fig. 6.

\section{CONCLUSION}

Cobalt doped Carbon nanotube is prepared by chemical method. In this method CNT's are prepared by treating egg albumin with $1.0 \mathrm{~N}$ Cobalt salt, prepared by $\mathrm{AR}$ grade techniques in alcoholic medium. The IR, NMR and X-RD predicted the lysine cobalt complex. The AFM and DLS confirmed the nano structure of carbon nanotubes. These carbon nanotubes are formed to exhibit electrical conductivity. This shows the presence of unpaired electron. It is also predicted to exhibit Para-magnetism.

\section{ACKNOWLEDGMENT}

Authors are thankful to the MH-HRD, Govt. of India for financial support and to the Director, IIT, ROORKEE, and India for providing spectral and analytical data. Authors are grateful to Divyanshu Prakash, Mr. Nishant Singh Rana and Dr. Ajit Joshi, HOD, Departments of Chemistry, Mewar University, India for valuable contribution and supports for research work.

\section{REFERENCE}

[1] K. Banerjee, "What are carbon nanotubes,"ACM/SIGDA ENewsletter, no. 22, September 2006.

[2] S. Iijima, "Helical microtubules of graphitic carbon," Nature, vol. 354, pp. 56-58, 1991.

[3] M. Ratnar et al., Nanotechnology a Gentle Introduction to Next Big Idea, Prentice Hall, 2003.

[4] S. B. Meo and R. Andrews. "Carbon nanotubes: synthesis, properties, and application," Crit. Rev.Solid State Mater. Sci., vol. 26, no. 3, pp. 145-249, 2001.

[5] G. Cao, Nanostructures and Nanomaterials: Synthesis, Properties and Application, Imperial College Press.

[6] M. S. Dresselhaus, G. Dresselhaus, and P. Avouris, Carbon Nanotubes: Synthesis, Structure, Properties and Applications, Springer-Verlag, 2000

[7] D. A. King, J. Am. Chem. Soc., vol. 122, pp. 10610-10614, 2000.

[8] J. Parker, "Gray level thresholding in badly illuminated images," IEEE Trans. on Pattern Analysis and Machine Intelligence, vol. 13, no. 8, pp. 813-819, 1999

[9] R. R. L. D. Oliveira et al., "Measurement of the nanoscale roughness by atomic force microscopy: basic principles and applications," Federal University of São Carlos, 2001.

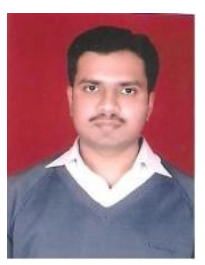

Sarvesh Kumar Shailesh was born on March 25, 1978 in Vaishali district of Bihar, India. Currently he is working as astt. professor at Mewar University in Chittorgarh, Rajasthan. He has submitted his D.phil. thesis on nanotechnology at the LNMU Darbhanga, Bihar. His research area is on Carbon nanotubes, composite materials, organic light emitting diode and quantum dot. He has published 5 international papers 
and attended 6 national and International conferences. He is member of Indian chemical society.

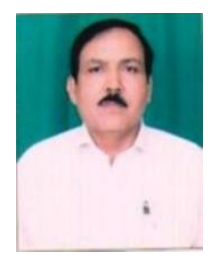

B. Tiwari is currently working as a director in DSITM Gaziabad. He has completed his D.phil. degree from B.

R. Ambedkar Bihar university on coordination chemistry and M.tech. from MVU. Currently his 3 students awarded D.phil. degree and two M.phil. degree and 15 students are pursuing Ph.D. under his supervision. His research area is on carbon nanotubes, composite materials, organic light emitting diode and quantum dot. He has published 35 research papers in international journals and attended 13 national and international conferences.
Avinash Kumar Roy has completed his master degree from Indian School of Mines Dhandad, Jharkhand. Currently he is pursuing his research on quantum dots. 